Helbig, Marko; Grießbach, Gert; Schack, Bärbel:

Objektorientierte Programmierung von Methoden der zeitvarianten Bispektralanalyse in der Biosignalverarbeitung

Zuerst erschienen in: Biomedizinische Technik = Biomedical Engineering. Berlin [u.a.] : de Gruyter. - 45 (2000), S1, S. 198-199.

Erstveröffentlichung: $\quad 2000$

Datum Digitalisierung: 2009-07-17

ISSN (online): $\quad$ 1862-278X

ISSN (print): $\quad$ 0013-5585

DOI: $\quad$ 10.1515/bmte.2000.45.s1.198

[Zuletzt gesehen: 2019-08-20]

„Im Rahmen der hochschulweiten Open-Access-Strategie für die Zweitveröffentlichung identifiziert durch die Universitätsbibliothek IImenau."

"Within the academic Open Access Strategy identified for deposition by IImenau University Library."

„Dieser Beitrag ist mit Zustimmung des Rechteinhabers aufgrund einer (DFG-geförderten) Allianz- bzw. Nationallizenz frei

zugänglich."

"This publication is with permission of the rights owner freely accessible due to an Alliance licence and a national licence

(funded by the DFG, German Research Foundation) respectively."

\section{DFG}

Nationallizenzen 


\title{
OBJEKTORIENTIERTE PROGRAMMIERUNG VON METHODEN DER ZEITVARIANTEN BISPEKTRALANALYSE IN DER BIOSIGNALVERAR- BEITUNG
}

\author{
M. Helbig ${ }^{1}$, G. Grießbach ${ }^{1}$, B. Schack ${ }^{2}$ \\ ${ }^{1}$ Institut f. Biomed. Technik u. Informatik, TU Ilmenau, Deutschland \\ ${ }^{2}$ Institut f. Med. Statistik, Informatik u. Dokumentation, FSU Jena, Deutschland \\ Marko.Helbig@informatik.tu-ilmenau.de
}

\section{EINLEITUNG}

Die Notwendigkeit des Einsatzes zeitvarianter Methoden in der Bispektralanalyse liegt im zumeist nichtstationären Charakter biologischer Signale begründet, wodurch die Anwendung klassischer, intervallbezogener Verfahren nur begrenzt möglich ist. Die trifft besonders auf transient auftretende quadratische Phasenkopplungen zu. Eine neue Methode auf der Basis der adaptiv rekursiven Schätzung der Kumulanten III. Ordnung wurde entwickelt und in [1] vorgestellt.

Bei der Entwicklung moderner Verfahren der Biosignalverarbeitung rückt neben der Bedeutung der signalanalytischen Merkmale die Effizienz ihrer Implementierung immer mehr in den Vordergrund. In der vorliegenden Arbeit wird die Umsetzung mit Hilfe objektorientierter Programmierung vorgestellt.

\section{METHODEN}

\section{Adaptive Kumulantenschätzung}

Grundlagen zu adaptiv rekursiven Schätzverfahren wurden u. a. in [2] beschrieben. Darin wurde von Grießbach erstmals ein Verfahren zur adaptiv rekursiven Schätzung der Korrelationsfunktion $\mathbf{K}^{\mathrm{C}}$ der Zeitreihen $X=\left\{x_{k}\right\}_{k=0,1, \ldots .}$ und $Y=\left\{y_{k}\right\}_{k=0,1, \ldots .}$ vorgestellt (1).

$\mathbf{K}^{c}(X, Y)=\left[\mathbf{M}^{c}\left(X \otimes \mathbf{L}_{0}(Y)\right), \quad \ldots, \mathbf{M}^{c}\left(X \otimes \mathbf{L}_{N}(Y)\right)\right](1)$

Dabei stellen $\mathbf{M}^{\mathfrak{c}}$ den adaptiv rekursiven Mittelwert, $\mathbf{L}_{\mathbf{k}}$ den Lagoperator und $\mathbf{c}, 0<\mathrm{c}<1$, die Adaptionsvariable dar (2).

$$
\begin{array}{ll}
M^{c}: & M_{0}=m_{0} \\
& M_{k+1}=M_{k}+c \cdot\left(x_{k+1}-M_{k}\right) \\
\mathbf{L}_{k}: & L_{k}\left(\left\{x_{j}\right\}_{j=0,1, \ldots .}\right)=\left\{x_{j+k}\right\}_{j=0,1, \ldots}
\end{array}
$$

Die Kovarianz ist die Kumulante II. Ordnung. Die indirekte Schätzung des Bispektrums beruht dagegen auf der Fouriertransformation der Kumulanten III. Ordnung. Auf der Basis dieses Zusammenhangs und aufbauend auf (1) wurde ein Algorithmus entwickelt, der es ermöglicht, auch die Kumulanten III. Ordnung und somit das Bispektrum zeitvariant zu schätzen (3).

$$
\operatorname{CUM}^{c}(X, Y, Z)=\left[\begin{array}{ccc}
\mathbf{M}^{c}\left(X \otimes \mathbf{L}_{0}(Y) \otimes \mathbf{L}_{0}(z)\right), & \ldots & , \mathbf{M}^{c}\left(X \otimes \mathbf{L}_{0}(Y) \otimes \mathbf{L}_{N}(Z)\right) \\
\mathbf{M}^{c}\left(X \otimes \mathbf{L}_{1}(Y) \otimes \mathbf{L}_{0}(z)\right), & \ldots & , \mathbf{M}^{c}\left(X \otimes \mathbf{L}_{1}(Y) \otimes \mathbf{L}_{N}(z)\right) \\
& \ldots & \\
\mathbf{M}^{c}\left(X \otimes \mathbf{L}_{N-1}(Y) \otimes \mathbf{L}_{0}(Z)\right) & \ldots & , \mathbf{M}^{c}\left(X \otimes \mathbf{L}_{N-1}(Y) \otimes \mathbf{L}_{N}(Z)\right) \\
\mathbf{M}^{c}\left(X \otimes \mathbf{L}_{N}(Y) \otimes \mathbf{L}_{0}(Z)\right) & \ldots & , \mathbf{M}^{c}\left(X \otimes \mathbf{L}_{N}(Y) \otimes \mathbf{L}_{N}(z)\right)
\end{array}\right]
$$

Eine Quantifizierung der Stärke quadratischer Phasenkopplungen setzt jedoch Signalamplitudenunabhängigkeit voraus, die mittels Bikohärenz und Phasenbikohärenz gegeben ist. Die ebenfalls zeitvariante Realisierung dieser Größen erfordert weitere an die Kumulantenschätzung anschließende mehrdimensionale Adaptionssteuerungen.

\section{Objektorientierte Programmierung}

Adaptiv rekursive Schätzverfahren bieten sich aufgrund ihrer Struktur an, mit Hilfe objektorientierter Programmiertechnologien implementiert zu werden. Abb. 1 zeigt eine Übersicht der wesentlichen Klassen, die diese Konzeption widerspiegeln. Die Darstellung erfolgt in UML-Notation.

Die abstrakte Klasse TAdaptBasisClass stellt den Ursprung der ganzen Klassenhierachie dar, von der selbst keine Objekte erzeugt werden können, in der aber bereits allgemeine, alle adaptiven Verfahren kennzeichnende Attribute und Methoden gekapselt werden.

Alle aus dieser Klasse abgeleiteten Klassen bilden die Implementierung adaptiver Grundelemente, so z.B. die adaptiven Formen des Mittelwertes oder des 2. statistischen Momentes. In ihnen wird die Methode Adapt überschrieben, wodurch der jeweilige in der abgeleiteten Klasse zugrunde liegende Schätzalgorithmus realisiert wird.

Die charakteristischen Merkmale bezüglich Schätzgüte und Dynamik werden maßgeblich durch die Adaptionsparameter (im einfachsten Fall eine Adaptionskonstante) festgelegt. Diese Parameter müssen zur Anpassung an zeitliche, z. T. sprunghafte Änderungen der Signaleigenschaften ebenso zeitvariant sein. 

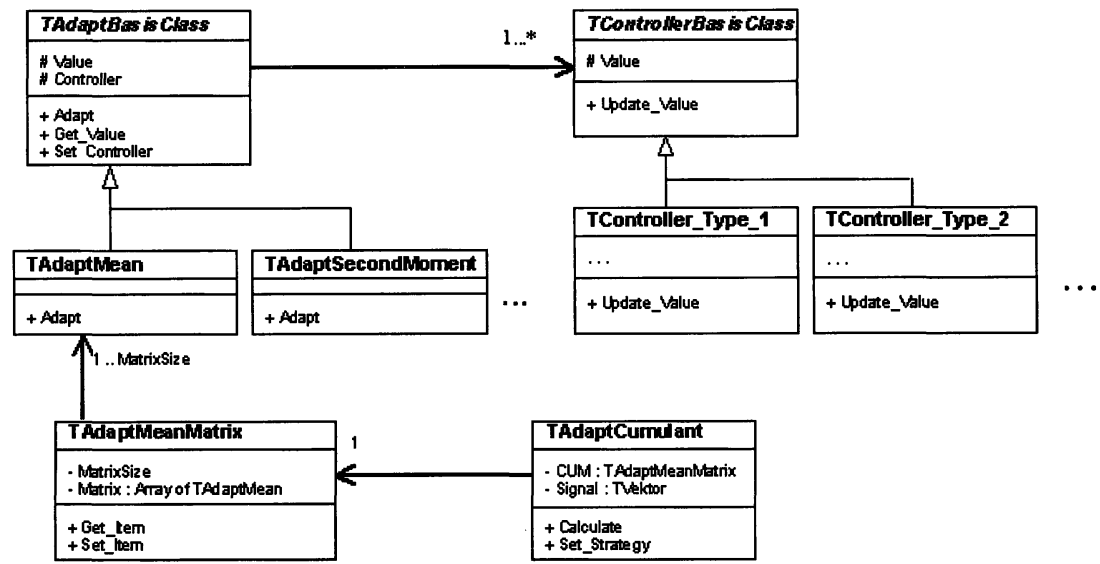

Abb. $1:$ Klassenstruktur

Diese Anpassungsoptimierungen werden mit Hilfe sog. Controller realisiert. In analoger Weise wird dazu mit TControllerBasisClass eine abstrakte Basisklasse bereitgestellt. Alle davon abgeleiteten Klassen verfolgen eigene Adaptionsstrategien.

Die Aufgaben der modernen Signalverarbeitung erfordern allerdings derartige Anpassungen in viel komplexeren Strukturen, wie sie z. B. die Kumulanten III. Ordnung darstellen. Prinzipiell lassen sich derartige komplexe Strukturen durch Aggregation einzelner adaptiver Grundelemente erzeugen. In [3] werden drei wesentliche Aggregationsformen dargestellt: arithmetische Verknüpfung, Verknüpfung innerhalb von

Vektoren sowie innerhalb von Matrizen. Einen typischen Anwendungsfall der Aggregation in Form von Matrizen stellt die adaptive Berechnung der Kumulanten III. Ordnung dar. Folgerichtig besitzt die Klasse TCumulant kein Array von Gleitkommazahlen, sondern eine Matrix bestehend aus TAdaptMeanObjekten, wobei jedes Objekt eine eigene Adaptionssteuerung (Controller) besitzt.

\section{ERGEBNISSE}

Die vorgestellten Methoden werden u. a. bei der Analyse von EEG-Burst-Mustern sedierter Patienten mit Schädelhirntrauma eingesetzt. Ziel der Untersuchungen ist die Bestimmung und Steuerung der Sedierungstiefe auf der Basis bispektraler Parameter der Burst-Muster. Es kann zum einen gezeigt werden, daß quadratische Phasenkopplungen während des Burstsauftritts stärker in Erscheinung treten als zu Beginn der Sedierung. Zum anderen kann der Zeitverlauf von Biamplitude und Bikohärenz innerhalb des Burstauftritts präzisiert werden.

Besonders bei der Weiterentwicklung der Algorithmen zur Steuerung der Adaptionsparameter wird die durchgesetzte Modularität von Vorteil sein.
Ebenso sind neue Parameter auf dieser Basis leicht $\mathrm{zu}$ implementieren.

\section{ZUSAMMENFASSUNG}

In der vorliegenden Arbeit wird eine Möglichkeit vorgestellt, neue Verfahren der digitalen Signalverarbeitung, in diesem Falle Methoden der zeitvarianten Bispektralanalyse, mit Hilfe der objektorientierten Programmierung effizient zu implementieren. Diese Technologien zeichnen sich bei konsequenter Durchsetzung ihrer Prinzipien durch ein hohes $\mathrm{Ma} \beta$ an Qualität der entstandenen Softwarepakete sowie durch effektive Wiederverwendbarkeit aus.

\section{LITERATURHINWEISE}

[1] Helbig, M., Grießbach, G., Schack,B., Witte, H.: Application of time-variant bispectrum in biosignal analysis, European Medical \& Biological Engineering Conference EMBEC'99, Vienna,, 1999, S. 392393

[2] Grießbach, G., Grießbach, U.: Adaptive Korrelationsverfahren in der Biosignalanalyse. In: Muche, R., Büchele, G., Harder, D., Gaus, W. (Hrsg.), Medizinische Informatik, Biometrie und Epidemiologie (82), MMV Medizin Verlag München, 1997, S. 8084

[3] Steuer, D., Bolten J., Grießbach G.: Effiziente Gestaltung komplexer adaptiver Schätzalgorithmen durch objektorientierte Programmierung, Biomed. Technik (1999, Erg.-Bd. 3), 1999, S. 27-31.

Die Arbeit wurde gefördert durch die DFG-Projekte GR 1555/2-1 und WI 1166/2-1. 